\title{
Spontaneous Rupture of Fibrolamellar Variant Hepatocellular Carcinoma
}

\author{
Abhishek D. Polavarapu ${ }^{\mathrm{a}}$, Moiz Ahmed ${ }^{\mathrm{b}}$, Ghassan Samaha ${ }^{\mathrm{a}, \mathrm{c}}$, \\ Chanudi Kashmalee Weerasinghe ${ }^{\mathrm{a}}$, Liliane Deeb ${ }^{\mathrm{a}}$, \\ Alisa Sokoloff ${ }^{a}$
}

\begin{abstract}
Fibrolamellar hepatocellular carcinoma (FL-HCC) is a unique variant of hepatocellular carcinoma. The majority of cases present with nonspecific symptoms like vague abdominal pain, weight loss and fatigue. Ruptured FL-HCC occurs rarely and mortality in the acute phase is very high. We report a rare case of a ruptured FL-HCC successfully managed with transarterial embolization for hemostasis. A 37-year-old male previously in good health presented with a severe, sharp epigastric pain that started $1 \mathrm{~h}$ prior to the presentation. He denied trauma, fever, nausea, vomiting, or diarrhea. Tenderness in the epigastrium was noted, with no palpable masses, guarding or rigidity. His blood pressure and pulse were 159/105 mm Hg and 105 beats/ min. Platelets and coagulation parameters were within normal limits; transaminases were elevated. Abdominal computed tomography (CT) scan with contrast revealed an $8 \mathrm{~cm}$ lobulated mass with central hypodensity in the left hepatic lobe with perilesional blood and free pelvic fluid, indicating tumor rupture. CT angiography showed tumor neovascularization from a branch of the left hepatic artery which was embolized using transarterial gelfoam. Liver magnetic resonance imaging (MRI) and biopsy were consistent with fibrolamellar variant hepatocellular carcinoma. After 4 days, as the symptoms resolved, and the lab results were stable, patient was discharged and underwent a left hepatectomy 3 weeks later. FL-HCC occurs commonly in the left lobe of a young and non-cirrhotic liver. Typically, cross sectional imaging reveals a lobulated mass with well-defined margins, areas of hypervascularity and a central calcified scar. Histologic appearance is characterized by eosinophilic polygonal shaped cells separated by lamellar fibrosis. Surgical resection is the treatment of choice with better outcome when compared to conventional HCC. Disease recurrence after complete surgical resection is however high in the first 5 years. Tumors $>5 \mathrm{~cm}$ in size are at high risk for rupture with high mortality and recurrence rates secondary to significant spillage of tumor. While an emergency hepatectomy is preferred in unstable patients, those that are hemodynamically stable can undergo radiologic transarterial embolization for hemostasis followed by staged
\end{abstract}

Manuscript submitted January 24, 2019, accepted April 1, 2019

aStaten Island University Hospital, Staten Island, NY, USA

bElmhurst Hospital, Elmhurst, NY, USA

${ }^{c}$ Corresponding Author: Ghassan Samaha, Staten Island University Hospital, Staten Island, NY 10305, USA. Email: ghassansamaha19@gmail.com

doi: https://doi.org/10.14740/gr1148 hepatectomy.

Keywords: Fibrolamellar carcinoma; Hepatocellular carcinoma; Tumor rupture; Liver neoplasm

\section{Introduction}

Fibrolamellar carcinoma (FLC) is a rare variant of hepatocellular carcinoma (HCC) that usually occurs in younger individuals without underlying liver disease. These patients typically present with chronic, nonspecific symptoms, such as dull abdominal pain, weight loss and fatigue. Here, we report on a spontaneous tumor rupture that was treated by transarterial embolization for hemostasis, followed by left hepatectomy. This is an extremely rare presentation of FLC that has been observed in only one case thus far.

\section{Case Report}

A 37-year-old male with no significant medical, surgical or travel history presented to our emergency room, complaining of severe, sharp epigastric and left upper quadrant pain that started $1 \mathrm{~h}$ prior to presentation. He reported no prior trauma, fever, nausea, vomiting, diarrhea or constipation. Physical examination revealed tenderness in the epigastric and left upper quadrants with no palpable masses, guarding or rigidity. His vital signs were stable, with a blood pressure of $159 / 105 \mathrm{~mm}$ $\mathrm{Hg}$ and heart rate of 105 beats/min. Laboratory results demonstrated a hemoglobin of $12 \mathrm{~g} / \mathrm{dL}$, white blood cell count of $11,000 / \mu \mathrm{L}$ and elevated transaminases (aspartate aminotransferase (AST) of $214 \mathrm{IU} / \mathrm{L}$, and alanine aminotransferase (ALT) of $138 \mathrm{IU} / \mathrm{L}$ ), alkaline phosphatase (ALP) of $98 \mathrm{IU} / \mathrm{L}$, and total bilirubin of $1.5 \mathrm{mg} / \mathrm{dL}$. Platelets count and coagulation parameters were within normal limits. An abdominal computerized axial tomography (CAT) scan with intravenous contrast revealed an $8 \times 7 \mathrm{~cm}$ heterogeneous lobulated mass with central hypodensity in the left hepatic lobe with perilesional blood and minimal high-density free pelvic fluid, indicating tumor rupture (Fig. 1). To confirm the diagnosis, the patient underwent computed tomography (CT) angiography, which demonstrated tumor neovascularization from a branch of the left 

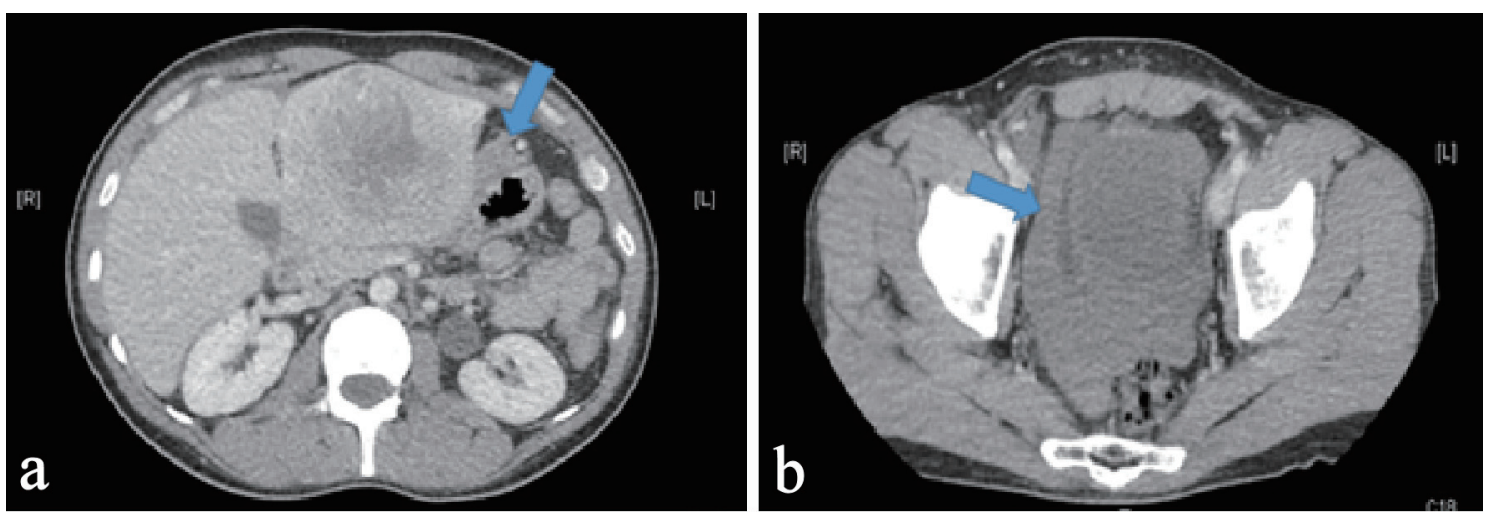

Figure 1. Axial section of CT abdomen and pelvis with intravenous contrast. (a) An $8 \times 7 \mathrm{~cm}$ heterogeneous lobulated mass with central hypodensity in the left hepatic lobe with arrow pointing perilesional blood concerning for tumor rupture. (b) An arrow pointing hyperdensity fluid surrounding urinary bladder likely blood from tumor rupture.

hepatic artery that was successfully embolized using transarterial gelfoam. For further tumor evaluation, magnetic resonance imaging (MRI) of abdomen with liver protocol was performed, revealing a heterogeneously enhancing left hepatic mass $(7.7$ $\mathrm{cm}$ in diameter) with central non-enhancing scar tissue compatible with fibrolamellar variant HCC (Fig. 2). Subsequent biopsy of liver mass confirmed this finding, as malignant hepatocytes filled with ample eosinophilic cytoplasm were observed. These tumor cells are arranged in cords separated by hyaline cartilage, consistent with FLC (Fig. 3). The patient's hepatitis serology was negative, with normal alpha-fetoprotein level. As the acute symptoms resolved, and the repeat laboratory tests including hemoglobin and liver biochemical tests yielded stable results, he was discharged from the hospital 4 days after admission. Three weeks later, a left hepatectomy was performed uneventfully. Fibrolamellar carcinoma diagnosis was confirmed as well after left hepatectomy. Resected specimen contained a well circumscribed lobulated tumor of $11 \times 8 \times$ $7 \mathrm{~cm}$ with margins free of tumor. Histopathological diagnosis

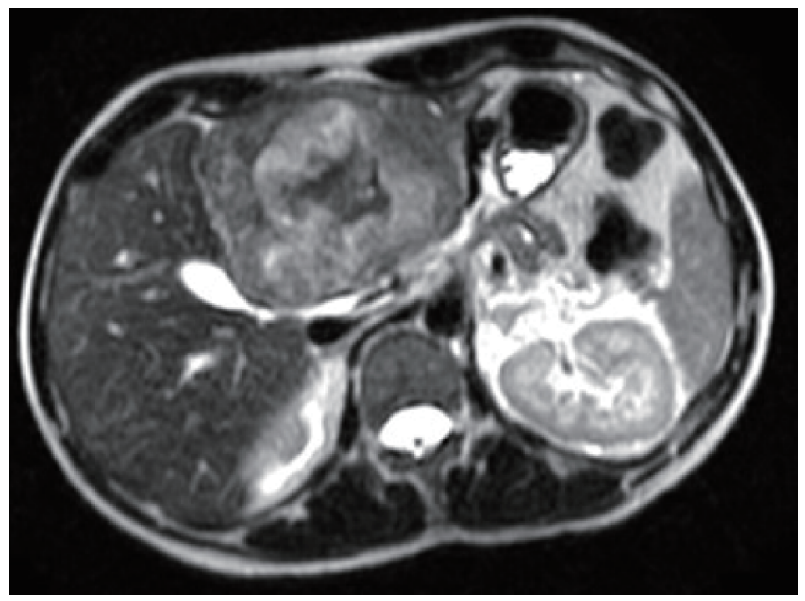

Figure 2. MRI of the abdomen with liver protocol post embolization showing a $7.7 \times 6.6 \mathrm{~cm}$ well circumscribed expansile lesion in left lobe of liver with global hypo T2 signal intensity with areas of hyper T2 signal intensities as well as central stellate shape scar of low T2 signa intensity. was also consistent with FHC. Focal hemorrhage and foci of necrosis were present, likely representing prior area of bleeding from tumor rupture.

\section{Discussion}

Fibrolamellar carcinoma (FLC) is an extremely rare and distinct variant of primary liver cancer categorized by World Health Organization under hepatocellular epithelial tumors [1]. It accounts for $1-9 \%$ of all liver cancers and, as in our case, typically occurs in young adults around the age of 30 years, with no sex predilection [2]. According to a recent study, however, the disease prevalence peaks again in older adults, aged 60 - 69 years, with a predilection for males [3].

Unlike hepatocellular carcinoma (HCC), FLC is diagnosed in patients with an apparently normal non-cirrhotic liver, as seen in our case [4]. Given its rarity, the mechanism of FLC tumorigenesis remains unknown. However, Honeyman et al discovered the presence of a mutation in DNAJB1-PRKACA in virtually all FLC cases, indicating that the fusion protein is likely the primary driver of FLC [5].

As in majority of FLC cases, patients present with nonspecific clinical symptoms, including weight loss, fatigue, and abdominal pain, a mass lesion is usually only revealed upon further detailed examination [4]. Given that some patients are asymptomatic, FLC may be discovered incidentally [4]. While the physical examination is often within normal limits, when present, common physical findings include a palpable abdominal mass or hepatomegaly with or without tenderness in the right upper quadrant [6]. Other rare presentations noted in pertinent literature include jaundice due to biliary obstruction, fulminant liver failure, recurrent deep vein thrombosis, encephalopathy, thrombophlebitis of the lower extremity, anemia, ascites, hypoglycemia, and gynecomastia in males [4]. Spontaneous tumor rupture as an initial presentation is very rare. Clinically, 66$100 \%$ of these patients present with acute signs and symptoms of peritonitis, including abdominal pain and tenderness [7]. In $33-90 \%$ of the cases, the presenting symptom is shock [7].

The laboratory tests, including liver biochemical and functional tests, were usually normal, but the results can be mildly 

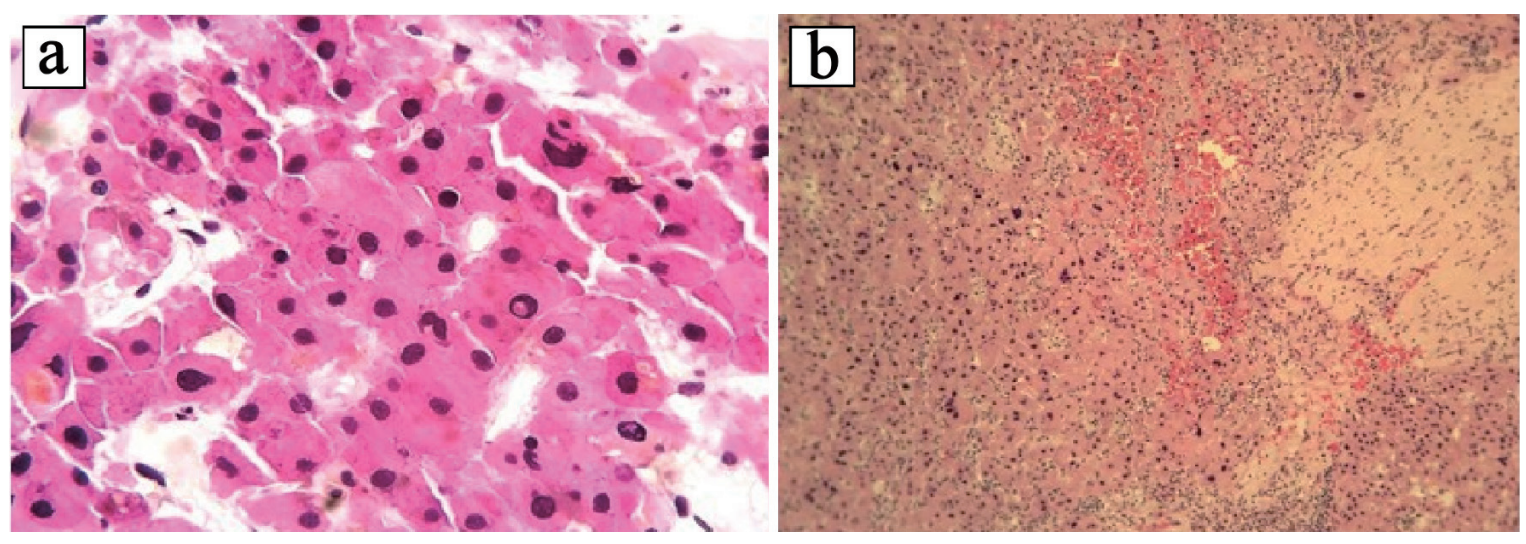

Figure 3. Histopathologic examination of hematoxylin and eosin (H\&E) stained liver biopsy specimen. (a) Tumor cells have hepatocellular appearance: large polygonal cells, ample eosinophilic cytoplasm, with focal bile pigment, marked nuclear atypia, and intranuclear pseudoinclusions. (b) Tumor cells are separated by hyaline collagen.

elevated is some cases. Unlike in traditional HCC, alpha fetoprotein levels are predominately normal in patients with FLC, whereas beta human chorionic gonadotropin can sometimes be elevated [8].

FLC diagnosis is typically guided by clinical presentation and imaging studies. On ultrasound, FLC presents as a well-defined mass that has heterogeneous echogenicity [4]. However, CT scan or MRI is required to differentiate FLC from other mass-forming lesions of the liver. CT scans with intravenous contrast typically reveal a large well-defined mass with a lobulated outline, heterogeneous hyperattenuation, and a central stellate scar (in $65-75 \%$ of patients) [9]. As in our case, the scar is identified as the central hypodensity in the image. When MRI is performed, FLC tumors usually appear as hypointense and hyperintense in T1- and T2-weighted images, respectively, with a fibrous central scar that remains hypointense in both imaging modes [9]. When gadolinium contrast agent is used during MRI, heterogeneous enhancement can be achieved [10]. In our patient, along with these typical features, we found evidence of free pelvic and perilesional high-density fluid, suggestive of intraperitoneal hemorrhage due to tumor rupture. Spontaneous tumor rupture is one of the most fatal complications of hepatic tumors occurring in about $3-26 \%$ of all such cases [7]. Consequently, mortality in the acute phase is very high, ranging from $32 \%$ to $67 \%$ [7]. Although tumor rupture pathogenesis remains insufficiently understood, it is thought to occur due to the rapid tumor expansion resulting in a high intratumoral pressure [7]. This leads to vascular congestion and central necrosis, and ultimately rupture. Based on the cases diagnosed to date, high propensity to rupture is associated with tumor protrusion from the liver surface, tumor size $>$ $5 \mathrm{~cm}$, vascular thrombus and extrahepatic invasion [11].

If cross-sectional imaging is insufficient for a definitive FLC diagnosis, tissue biopsy is recommended. As fine needle aspiration has low yield, either percutaneous core biopsy or open biopsy via laparoscopy is the preferred technique [4]. Microscopically, FLC is characterized by large polygonal or spindle-shaped tumor cells with deeply eosinophilic cytoplasm due to abundant mitochondria and prominent nuclei arranged in cords surrounded by lamellated collagen fibers [9]. Immuno- histochemical staining of FLC reveals some similarities with $\mathrm{HCC}$, including tumor tissue staining positive for hepatocyte paraffin 1. However, unlike HCC, FLC often stains strongly for CK7 and epithelial membrane antigen, which are characteristic of biliary differentiation and serve as markers of hepatic differentiation (CK19 and EpCAM) [9]. In addition, unlike most HCC, FLC stains negative for alpha fetoprotein. In our case, percutaneous core biopsy of liver mass revealed not only these typical microscopic features, but also positive staining for $\mathrm{CK}$ and $\mathrm{EpCAM}$, thereby confirming the initial diagnosis.

When feasible, surgery is the only potential curative treatment option. Empirical evidence indicates that $70-75 \%$ of such carcinomas are amenable to complete resection, aiming for negative margins with adequate lymph node dissection [6]. Given that most FLC patients have no underlying liver disease, a substantial amount of the liver parenchyma can be removed owing to the strong regenerative potential of the remaining liver. Five-year overall survival rates following aggressive resection range from $58 \%$ to $82 \%$ [6]. However, outcomes can be highly variable, as $50-100 \%$ relapse rate is reported following even radical surgery, whereby a 20 - to 48 -month median recurrencefree survival has been reported [12]. Thus recurrence following FLC resection is also relatively common, ranging from $33 \%$ to $100 \%$ [13]. Due to the high recurrence rate, diligent postoperative surveillance is indicated. The follow-up protocol for the first 2 - 3 years postoperatively should include CT and serum vitamin B12-binding protein levels every 3 - 6 months. Elevated vitamin B12 binding protein has been reported as a specific marker of FLC and is thought to be due to production or modification of the binding protein transcobalamin I by the tumor [14].

At the opposite end of the spectrum are the $20-25 \%$ of patients who have categorically unresectable disease due to multifocal metastases, extensive nodal spread, and/or major vessel involvement [6]. In such cases, $0-5 \% 5$-year survival rate, with a median survival of 12 months or less, is reported [13]. Systemic therapy is the only recommended option for these patients, albeit with limited effectiveness. Available case reports suggest that platinum-based regimens, such as cisplatin, epirubicin, and fluorouracil (FU) combination could offer some benefits [15]. 
The unique aspect of our case was acute tumor rupture as the presenting symptom. Hemostasis is the primary objective in acute management of a ruptured hepatic tumor. In unstable patients, an emergency hepatectomy is recommended. On the other hand, in hemodynamically stable cases, a two-stage approach involving radiologic transarterial embolization (TAE) for hemostasis, followed by staged hepatectomy, is preferred as this approach is associated with lower in-hospital mortality rate (0-9\% vs. 16-100\% reported for single-stage emergency liver resection) [7]. The serum bilirubin level, presence of shock on hospital admission, and pre-rupture disease state are important prognostic factors for predicting survival in the acute phase [7]. As our patient's vital signs were stable, this was indicative of limited intraperitoneal hemorrhage due to tumor rupture, he underwent TAE for hemostasis followed by staged hepatectomy. The only contraindication for TAE is a complete occlusion of the main portal vein by the tumor, which may cause hepatic infarction. The American Joint Committee on Cancer/Union for International Cancer Control (AJCC/UICC) classifies all ruptured HCCs as stage T4 in current TNM staging [16].

Due to the limited number of reported cases, it is difficult to establish the long-term outcomes of ruptured FLC tumor with any degree of certainty. In such patients, there is significant spillage of tumor secondary to the rupture, due to which cancer dissemination occurs with subsequent disease recurrence, ultimately leading to death. As previously noted, this is only the second report of a case where a patient presented with spontaneous rupture of FLC. However, in the previous case [10], it was managed by an emergency hepatectomy as the patient was unstable; but in our case, as the patient was stable, a two-stage approach involving radiologic TAE for hemostasis followed by staged hepatectomy was done.

\section{Acknowledgments}

Acknowledgment for the Pathology Department for providing the surgical biopsy slides.

\section{Financial Disclosure}

None to disclose.

\section{Conflict of Interest}

None to disclose by any of the contributing authors.

\section{Informed Consent}

No patient identifiers, verbal informed consent obtained.

\section{Author Contributions}

All mentioned authors had contributed in the writing and/or editing of the manuscript.

\section{References}

1. Bosman FT, Carneiro F, Hruban RH, Theise ND. WHO classification of tumours of the digestive system. World Health Organization; 2010.

2. National Cancer Institute D SRP. Cancer Statistics Branch Surveillance, Epidemiology, and End Results (SEER) Program. 2015. Available from: http://www.seer.cancer. gov.

3. Eggert T, McGlynn KA, Duffy A, Manns MP, Greten TF, Altekruse SF. Epidemiology of fibrolamellar hepatocellular carcinoma in the USA, 2000-10. Gut. 2013;62(11):1667-1668.

4. Kassahun WT. Contemporary management of fibrolamellar hepatocellular carcinoma: diagnosis, treatment, outcome, prognostic factors, and recent developments. World J Surg Oncol. 2016;14(1):151.

5. Honeyman JN, Simon EP, Robine N, Chiaroni-Clarke R, Darcy DG, Lim, II, Gleason CE, et al. Detection of a recurrent DNAJB1-PRKACA chimeric transcript in fibrolamellar hepatocellular carcinoma. Science. 2014;343(6174):1010-1014.

6. Ang CS, Kelley RK, Choti MA, Cosgrove DP, Chou JF, Klimstra D, Torbenson MS, et al. Clinicopathologic characteristics and survival outcomes of patients with fibrolamellar carcinoma: data from the fibrolamellar carcinoma consortium. Gastrointest Cancer Res. 2013;6(1):39.

7. Lai EC, Lau WY. Spontaneous rupture of hepatocellular carcinoma: a systematic review. Arch Surg. 2006;141(2):191-198.

8. Riggle KM, Turnham R, Scott JD, Yeung RS, Riehle KJ. Fibrolamellar hepatocellular carcinoma: mechanistic distinction from adult hepatocellular carcinoma. Pediatr Blood Cancer. 2016;63(7):1163-1167.

9. Lafaro KJ, Pawlik TM. Fibrolamellar hepatocellular carcinoma: current clinical perspectives. J Hepatocell Carcinoma. 2015;2:151-157.

10. Minutolo V, Licciardello A, Arena M, Minutolo O, Lanteri R, Arena G. Surgical resection of ruptured fibrolamellar hepatocellular carcinoma. Case Rep Surg. 2013;2013:679565.

11. Zhu Q, Li J, Yan JJ, Huang L, Wu MC, Yan YQ. Predictors and clinical outcomes for spontaneous rupture of hepatocellular carcinoma. World J Gastroenterol. 2012;18(48):7302-7307.

12. Eggert T, McGlynn KA, Duffy A, Manns MP, Greten TF, Altekruse SF. Fibrolamellar hepatocellular carcinoma in the USA, 2000-2010: A detailed report on frequency, treatment and outcome based on the Surveillance, Epidemiology, and End Results database. United European Gastroenterol J. 2013;1(5):351-357.

13. Stipa F, Yoon SS, Liau KH, Fong Y, Jarnagin WR, D'Angelica M, Abou-Alfa G, et al. Outcome of patients with fibrolamellar hepatocellular carcinoma. Cancer. 2006;106(6):1331-1338. 
14. Maniaci V, Davidson BR, Rolles K, Dhillon AP, Hackshaw A, Begent RH, Meyer T. Fibrolamellar hepatocellular carcinoma: prolonged survival with multimodality therapy. Eur J Surg Oncol. 2009;35(6):617-621.

15. Bower M, Newlands ES, Habib N. Fibrolamellar hepatocellular carcinoma responsive to platinum-based com- bination chemotherapy. Clin Oncol (R Coll Radiol). 1996;8(5):331-333.

16. Edge SB, Compton CC. The American Joint Committee on Cancer: the 7th edition of the AJCC cancer staging manual and the future of TNM. Ann Surg Oncol. 2010;17(6):1471-1474. 